\title{
Phagocyte function and cytokine production in community acquired pneumonia
}

\author{
Khalid Moussa, Hilary J Michie, Ian A Cree, Ann C McCafferty, John H Winter, \\ D Paul Dhillon, Susan Stephens, Richard A Brown
}

\begin{abstract}
Background - It is possible that many deaths from pneumonia may involve the generation of inflammatory mediators and tissue damage by activated phagocytes. To test this hypothesis phagocyte function, plasma levels of interleukin 6 (IL-6), tumour necrosis factor alpha (TNF $\alpha)$, and soluble interleukin 2 receptor (IL-2R), disease severity, and outcome have been examined in 46 patients with community acquired pneumonia. Methods - Polymorphonuclear leucocyte (PMNL) and monocyte function were measured daily by chemiluminescence in these patients during the first week of admission, and cytokine levels were subsequently determined by ELISA. A series of 61 healthy individuals were used as a control group for the chemiluminescence results.

Results - There was evidence of phagocyte, particularly PMNL, activation on admission in $76 \%$ of the patients. Most patients $(86 \%)$ also had raised IL-2R levels on admission. IL-6 and unbound TNF $\alpha$ were present in $23 \%$ and $41 \%$ of patients at varying times during the course of the disease. There was little correlation between measurements of cytokine or phagocyte levels and outcome or indicators of disease severity, although this may be because of the small number of patients included in this preliminary study.

Conclusions - These results are consistent with the hypothesis that activated phagocyte function and raised levels of circulating cytokines may contribute to the pathogenesis of community acquired pneumonia. There are striking similarities in this respect between pneumonia, adult respiratory distress syndrome, and sepsis.
\end{abstract}

(Thorax 1994;49:107-111)

Community acquired pneumonia is a considerable problem in terms of its morbidity, mortality and use of hospital resources. ${ }^{12}$ It causes 10 times as many deaths in the UK as all other infectious diseases together, and the sub- group of patients who have pneumococcal bacteraemia have an even greater mortality which reaches $30 \%$ in some studies. ${ }^{23}$ In 1964 Austrian and Gold ${ }^{4}$ showed that the mortality curves of patients with pneumococcal pneumonia who did not receive antibiotics and those treated with penicillin diverged after four days. Intravenous penicillin eradicates viable pneumococci from sputum in a few hours, so it seems evident that the invading bacteria trigger a pathological process which leads inexorably to multiple organ failure and death. ${ }^{3}$

The involvement of phagocytes in pneumonia is well known. Infiltration of affected lung parenchyma by neutrophils occurs within a short time in response to infection, and is followed by an influx of monocytes. Killing of bacteria by antibiotic treatment is associated with resolution of this inflammatory response. However, some patients in whom resolution does not occur show pathological features which are similar to those seen in the adult respiratory distress syndrome (ARDS). Multiple organ failure is often present in patients with ARDS, as it is in patients with pneumonia who do not respond to treatment.

There is compelling evidence that mediator release from phagocytes is central to the endothelial cell damage which seems to be the hallmark of ARDS. ${ }^{5-9}$ The role of cytokines and, in particular, tumour necrosis factor alpha $(T N F \alpha)$ in this process has been closely investigated in ARDS and septic shock. ${ }^{10}$ Since phagocytes are potent producers of TNF $\alpha$, we have studied phagocyte function and circulating cytokine levels in patients with pneumonia to determine whether phagocyte function and cytokine levels are abnormal in these patients. We have recently reported preliminary results from this study, which show that many of the patients included in our study had circulating $T N F \alpha$ at some point during their stay in hospital. ${ }^{11}$

\section{Methods}

PATIENTS

Forty two consecutive admissions to King's Cross Hospital, Dundee with community acquired pneumonia were included in the study. Pneumonia was defined as the presence of new shadowing on a chest radiograph in 
conjunction with an appropriate clinical history and examination for which no other cause was found. Informed consent was obtained from all patients and the study was approved by the Tayside Ethical Committee. The average age of the patients was 60 years with a range of 13-87 years; 30 were men. Twelve patients had evidence of chronic obstructive airways disease and some form of pre-existing lung disease was present in a total of 16 patients. Of the 42 patients included in this sample, eight had pneumococcal infection proven by culture (five blood, three sputum), one had Klebsiella (sputum), one had Staphylococcus aureus, one had influenza B, and two had mycoplasma infection proven by serological testing. The aetiological agent was not established in 30 patients due, in part, to the loss of urine samples taken for assay of pneumococcal antigen. Six patients died. The stay in hospital of those who survived varied from 1-42 days (median 11 days), excluding one patient with social problems which prevented his discharge. Thirteen patients had received treatment from their general practitioner before admission. Consent was obtained from each patient or their relatives and $20 \mathrm{ml}$ blood was taken daily starting on the morning after admission; $10 \mathrm{ml}$ was taken into lithium heparin anticoagulant tubes for phagocyte assays, $5 \mathrm{ml}$ into EDTA tubes, and $5 \mathrm{ml}$ into a plain glass tube for serum collection. A further blood sample was taken from each survivor eight weeks after discharge for both phagocyte and cytokine assays. Clinical and other parameters collected for each patient included chest radiographic appearances, temperature, respiratory rate, presence or absence of confusion, blood pressure, arterial blood gases (if appropriate), white cell count, platelet count, lymphocyte subsets, complement level, renal and liver function.

HEALTHY CONTROLS

Sixty $\mathrm{ml}$ heparinised venous blood samples were obtained from 61 healthy volunteers aged 20-60 years (median 36 years; male:female ratio $1: 0.92$ ), the majority of whom were staff members of a local agricultural research insti- tute. Since the results of phagocyte function tests might be influenced by intercurrent viral infection or atopy, each subject was carefully questioned about their past and present medical history, drug treatment, atopy, and general health. All subjects were asked to report upper respiratory tract infections and other illnesses occurring in the week after blood donation. Those reporting such infections or illnesses were excluded from the control data set.

POLYMORPHONUCLEAR LEUCOCYTE (PMNL) AND MONOCYTE PREPARATION

PMNLs and monocytes were obtained by density centrifugation and their function assessed by lucigenin-enhanced chemiluminescence assay as previously described, with the following modifications. ${ }^{12-14}$ The cells were separated by layering anticoagulated venous blood over Lymphoprep (Nycomed UK Ltd, Birmingham, UK) and dense ficoll. The dense ficoll was prepared by mixing $21 \mathrm{~g}$ Ficoll 400 (Pharmacia Ltd, Milton Keynes, UK) dissolved in $150 \mathrm{ml}$ sterile distilled water with $53 \mathrm{~g}$ sodium diatrizoate (Sigma Chemical Co, Poole, UK) dissolved in $80 \mathrm{ml}$ sterile distilled water, and adjusting the specific gravity to $1 \cdot 119$. The chemiluminescence response to phorbol myristate acetate (PMA), serum opsonised zymosan (SOZ), zymosan and buffer (HBSS control) was measured in a Dynatech ML1000 luminometer using white plastic microtitre plates (Dynatech).

\section{CYTOKINE ASSAYS}

Assays for IL- 6 and TNF $\alpha$ were performed by enzyme linked immunosorbent assay (ELISA). IL-6 levels were measured using a commercially available kit (Medgenix Diagnostics, Fleurus, Belgium), while free (nonbound) $T N F \alpha$ was measured by an ELISA system developed by Engelberts et al. ${ }^{15}$ Soluble interleukin 2 receptor (IL-2R) in plasma was measured by ELISA (Cellfree, $T$ Cell Sciences, Cambridge, USA). Normal range data provided with both assays was used to determine whether results from patients with pneumonia were normal or abnormal.

\section{DATA ANALYSIS}

The results were analysed as profiles for individual patients and as grouped data for comparison of patients with control subjects. Linear regression analysis was used to examine the relation between the parameters measured, and the results were compared with a healthy control population using the KolmogorovSmirnov (KS) test. The 95th percentiles describing the control population chemiluminescence data were used to determine the number of patients with chemiluminescence responses above the normal range. Differences between data grouped according to prognosis and outcome were compared by the Wilcoxon rank sum test. 


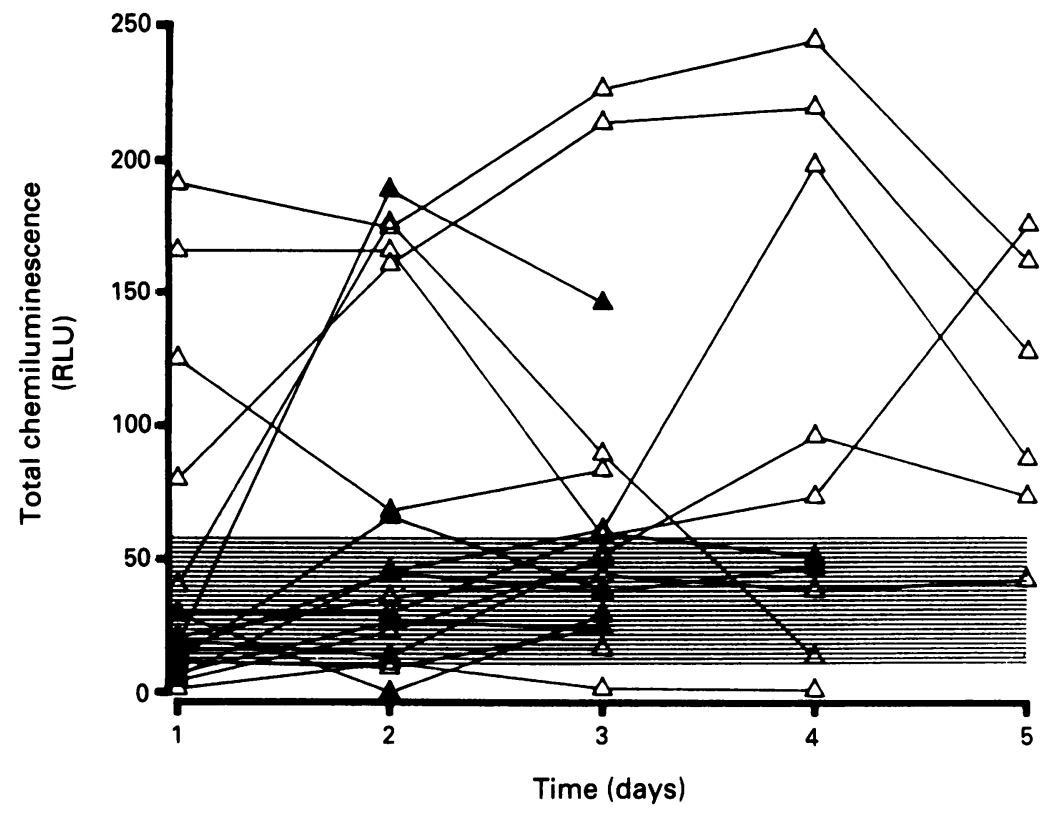

Figure 1 Phorbol ester-stimulated responses from polymorphonuclear leucocytes in patients for whom at least three consecutive daily blood samples were obtained. Open triangles = no treatment before admission, closed triangles = treatment before admission, shaded area $=$ normal (95th percentile) range.

\section{Results}

\section{PHAGOCYTE CHEMILUMINESCENCE}

The chemiluminescence results are summarised in the table. On admission the PMNLs from 32 patients $(76 \%)$ showed high background chemiluminescence production over the duration of the assay without added stimulant in comparison with a group of 61 healthy controls tested on a single occasion (KS test, $\mathrm{p}<0.0001)$. PMNLs from $30(71 \%)$ of the patients also produced a higher chemiluminescence response to stimulation by unopsonised zymosan than controls (KS test, $\mathrm{p}<0.0001$ ). The patients had raised PMNL chemiluminescence responses to PMA and SOZ in comparison with controls, although this was less significant than the non-specific responses noted above (KS test, $\mathrm{p}<0.01$ and $\mathrm{p}<0.002$ respectively). Although 20 patients $(48 \%)$ showed increased PMNL responses to PMA on admission, there were also six patients

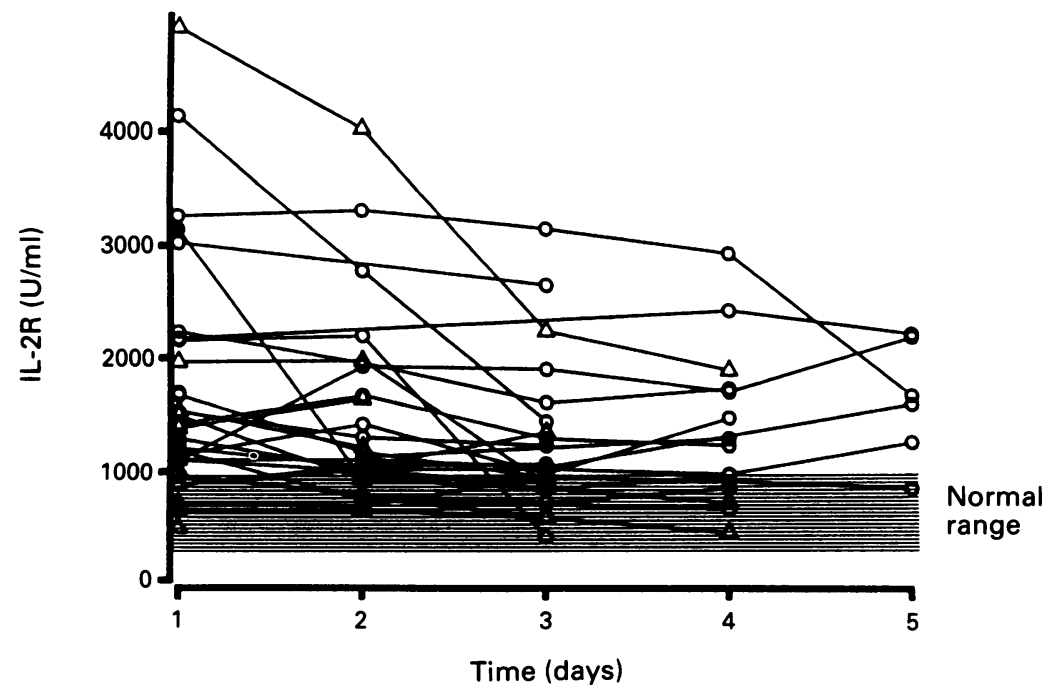

Figure 2 Soluble interleukin 2 receptor results from all patients included in the study over time in days following admission. Open triangles = treated cases, open circles $=$ untreated cases, solid symbols represent patients who died, shaded area $=$ normal range.
$(14 \%)$ whose responses were reduced in comparison with the normal range. In those patients who had not received treatment before admission (fig 1) the PMNL chemiluminescence response to PMA conformed to two patterns over the first week: (1) a high initial value followed by a slow decline to normal levels (6/20); and (2) a low or normal initial reading followed by a gradual rise $(14 / 20)$.

Monocyte chemiluminescence responses were also raised compared with healthy controls, with high initial responses to PMA in 20 patients $(48 \%, \mathrm{KS}$ test, $\mathrm{p}<0.05)$, to $\mathrm{SOZ}$ in 15 patients $(36 \%, \mathrm{NS})$, and to unopsonised zymosan in 14 patients $(33 \%, \mathrm{NS})$. However, as with PMNLs, the striking feature was the high unstimulated monocyte production of chemiluminescence by 30 patients in comparison with healthy controls $(71 \%$, KS test, $\mathrm{p}<0.0001)$. The monocyte chemiluminescence responses on admission showed good linear correlation with the initial PMNL chemiluminescence responses for both PMA $(r=0.778, \mathrm{p}<0.0001)$ and $\mathrm{SOZ}(r=0.704$, $\mathrm{p}<0.0001)$.

\section{CYTOKINES}

As we have previously reported ${ }^{11} 16$ of 39 patients $(41 \%)$ in whom $T N F \alpha$ measurements were obtained had free $\mathrm{TNF} \alpha$ in their plasma at some point during their illness, nine on admission and seven subsequently. Circulating $\mathrm{TNF} \alpha$ was present in two of the six patients who died. $T N F \alpha$ levels ranged from 10 to $354 \mathrm{pg} / \mathrm{ml}$ (median $76 \mathrm{pg} / \mathrm{ml}$ ). The number of days on which $\mathrm{TNF} \alpha$ was found in the plasma varied from one to four. All seven patients in whom circulating $\mathrm{TNF} \alpha$ was found after admission had not received antibiotics before admission.

Interleukin 6 was present in the plasma in only eight of 34 patients, five of whom also showed $\mathrm{TNF} \alpha$ at some stage during their illness. It preceded the appearance of TNF $\alpha$ in all but one of these five cases. IL-2R was raised on admission in 32 of 37 patients $(86 \%$, median $1286 \mathrm{U} / \mathrm{ml}$, range 483-4899 U/ml), but only five patients showed a rising IL-2R level during their hospital admission (fig 2).

\section{RELATION BETWEEN INDIVIDUAL} IMMUNOLOGICAL PARAMETERS

The relation between the laboratory measurements was difficult to assess since the timing and speed of pathogenetic events in the patients was unknown. In patients who were not treated with an antibiotic before admission to hospital, examination of the phagocyte results showed that these also tended to rise after treatment, and the IL-6 level rose before TNF $\alpha$ in three out of four patients in whom both cytokines were measured (fig 3 ).

RELATION BETWEEN IMMUNOLOGICAL AND CLINICAL PARAMETERS

The British Thoracic Society (BTS) identified patients with a poor prognosis as those having 


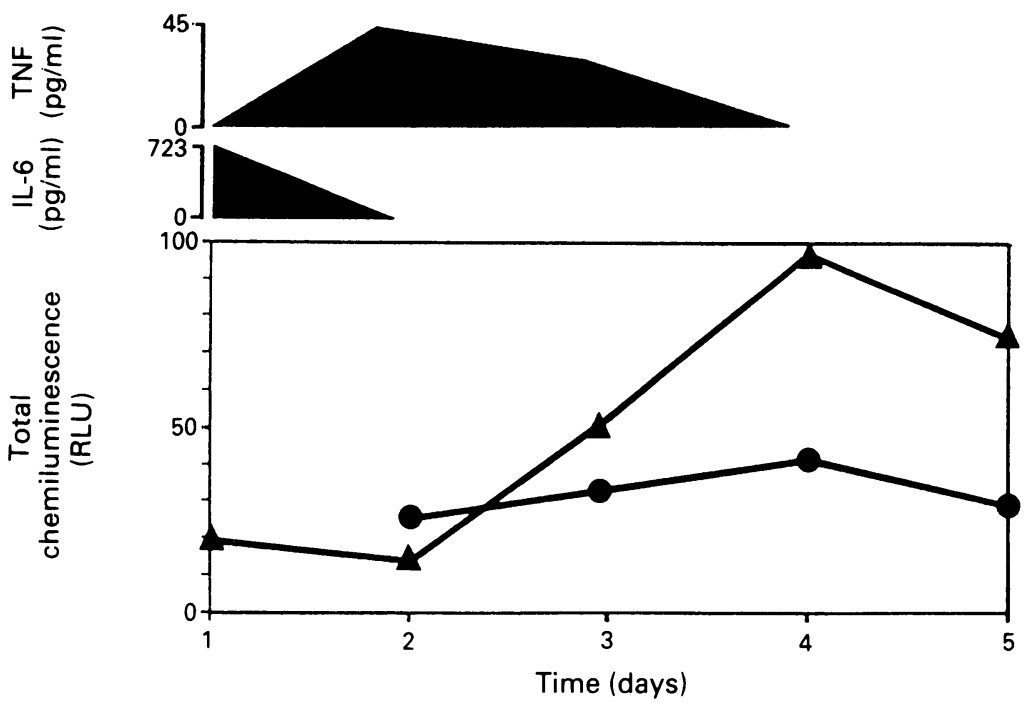

Figure 3 The course of pneumonia in one patient. Antibiotic treatment was given on admission and a rise in interleukin 6 was seen the next morning. A subsequent rise in the polymorphonuclear leucocyte chemiluminescence response to phorbol ester (PMA) is preceded by the appearance of $T N F \alpha$ in the peripheral blood.

a respiratory rate $>30 / \mathrm{min}$ on admission, a diastolic blood pressure $<60 \mathrm{~mm} \mathrm{Hg}$ on admission, and a urea concentration of $>7 \mathrm{mmol} / 1$ during admission. ${ }^{2}$ Fourteen $(33 \%)$ of the 42 patients in the present study were categorised on this basis as having a poor prognosis and the remainder as having a good prognosis. When the individual laboratory parameters were compared between these two groups, the poor prognostic group showed no significant differences in either the level of phagocytes or the other immunological parameters measured. However, the poor prognostic group did spend longer in hospital (Wilcoxon rank sum test, $\mathrm{p}<0.05)$. Other criteria found to be important in the BTS study included the white cell count, confusion, low arterial oxygen $(<8 \mathrm{kPa}$ or $60 \mathrm{~mm} \mathrm{Hg})$, and low serum albumin concentrations $(<35 \mathrm{~g} / \mathrm{l})$. The phagocyte chemiluminescence results and immunological investigations showed no relation to any of these clinical parameters. No significant differences in phagocyte activity or cytokine levels were found between those patients with and those without chronic obstructive airways disease.

Twelve patients were given antibiotic treatment before they were admitted and only one of these subsequently died. Twenty five patients received no treatment before admission and there was some uncertainty in the remaining five cases. No significant differences in immunological parameters were found between the previously treated and untreated patient groups.

\section{Discussion}

Phagocytes in patients with community acquired pneumonia are activated in comparison with healthy control subjects, as they are in ARDS $^{9}$ and other inflammatory disease states. ${ }^{16}$ Both PMNLs and monocytes produce more reactive oxygen species without stimulation than controls. These cells are also primed to produce larger responses than controls when stimulated with PMA or opsonised par- ticles. Their enhanced ability to respond to non-opsonised zymosan may reflect changes in the cell membrane which promote non-specific phagocytosis. ${ }^{1718}$

In agreement with a recent report, ${ }^{19}$ most patients had high levels of soluble IL-2R in their plasma on admission (fig 1), suggesting that they had substantial lymphocytemediated immune responses. Since it is usually assumed that pneumonia reflects a non-specific acute inflammatory response to infection, this finding emphasises the fact that potentially pathogen-specific immune responses are involved at an early stage.

One of the problems of studying pneumonia is that of knowing, with any degree of accuracy, when the infection started or the rate of progression of the illness. However, those patients who reached hospital without having received antibiotic treatment are a useful subgroup since they allow the effects of current treatment strategies on the host response to be evaluated. Many of those patients who were not treated before admission had low or normal phagocyte chemiluminescence on admission. As phagocyte chemiluminescence rises after admission and antibiotic treatment in this group of patients, it seems likely that it is not due to prior stimulation and phagocyte "fatigue." It is possible, however, that either the more activated cells in non-treated patients have been sequestered in the lung and are therefore not present in the peripheral blood, or that the phagocytes are being primed as a result of antibiotic treatment. These two explanations are not mutually exclusive, since a small proportion of activated phagocytes would not be detected by the phagocyte chemiluminescence method used, which averages the results from 25000 cells in each well of the microtitre plate.

Our original hypothesis was based on the similarity between fatal pneumonia and ARDS, in which TNF $\alpha$ and phagocyte activation have been shown to have a key role, probably through their interaction with endothelial cells. ${ }^{710} \mathrm{~A}$ high proportion of patients with pneumonia have circulating unbound $\mathrm{TNF} \alpha$ at some stage in their illness and some have IL-6, usually at an early stage in their admission. In septic shock IL-6 levels are usually high in the first 24 hours and then decline quickly.

Since both TNF $\alpha$ and IL-6 may be released in large quantities from macrophages, including lung macrophages, ${ }^{7}$ the relation between the release of these cytokines and alterations in phagocyte function is of particular interest. One would expect measurements of phagocyte function to rise at the same time as $T N F \alpha$ release, and this does occur (fig 2). It also appears that IL-6 release precedes evidence of systemic phagocyte priming.

Antibiotic treatment may have major effects on these mechanisms, since it is followed by an increase in phagocyte function and the appearance of circulating $T N F \alpha$ in the peripheral blood of at least some patients with pneumonia. This agrees with the old clinical observation that untreated lobar (pneumococcal) 
pneumonia resolved by crisis with high fever, in which endogenous pyrogens such as $\mathrm{TNF} \alpha$, IL-6, and IL-1 are known to play a major part. $\mathrm{TNF} \alpha$, lipopolysaccharide, IL-1 and other mediators act partly by promoting the expression of signals on the surface of the endothelial cell which promote PMNL adhesion. ${ }^{20}$ Thus our results are in agreement with the hypothesis that similar mechanisms may indeed operate in sepsis and pneumonia. Since IL-6 plays a major part in the acute phase response, its appearance in the plasma of patients with pneumonia may simply reflect early inflammatory events within the lung following the release of antigen from bacteria killed by antibiotic treatment within the first few hours of admission.

Too few deaths occurred during this study to make any firm statements about the clinical relevance of the changes we have observed. There was also little correlation between the immunological parameters measured and prognostic or other clinical parameters. However, it would be premature to conclude that they have no relevance, and the six deaths $(14 \%)$ in this series of patients illustrate graphically that the pathogenesis of pneumonia requires further study.

In view of the increasing evidence that $\mathrm{TNF} \alpha$ release and endothelial damage by adherent phagocytes leads to the development of ARDS, it seems likely that our inability to show clinical relevance simply reflects the complex situation present in patients with pneumonia. Studies of patients with ARDS have also shown poor correlation between $\mathrm{TNF} \alpha$ levels or phagocyte function and outcome. ${ }^{92122}$ It is becoming clear, however, that the timing of events is critical, and that mechanisms which downregulate inflammatory mechanisms and protect the endothelium may have as important a role as those which mediate damage. ${ }^{23}$ If this is the case, then it will prove difficult to predict which patients are at risk. Nevertheless, alteration of treatment regimens in community acquired pneumonia to avoid potentially injurious mediator release and phagocyte activation may be possible.

1 Woodhead MA, MacFarlane JT, McCracken JS, Rose DH, Finch RG. A prospective study of the aetiology and outcome of pneumonia in the community. Lancet 1987;i:671-4

2 British Thoracic Society Research Committee. Community-acquired pneumonia in adults in British hospitals in 1982-1983: a survey of aetiology, mortality, prognostic factors and outcome. $Q 7$ Med 1987;62:195-220.

3 Hook EW, Horton CA, Schaberg DR. Failure of intensive care unit support to influence mortality from pneumococcal bacteraemia. $\mathcal{F} A M A$ 1983;249:1055-7.

4 Austrian R, Gold J. Pneumococcal bacteremia with especial reference to bacteremic pneumococcal pneumonia. Ann Intern Med 1964;60:759-76.

5 Tracy KJ, Lowry SF, Cerami A. Cachectin/TNF $\alpha$ in septic shock and septic adult respiratory distress syndrome. $\mathrm{Am}$ Rev Respir Dis 1988;138:1377-9.

6 Rivkind AI, Siegel JH, Guadalupi P, Littleton M. Sequential patterns of eicosanoid, platelet, and neutrophil interactions in the evolution of the fulminant post-traumatic adult respiratory distress syndrome. Ann Surg adult respiratory

7 Swank DL, Moore SB. Roles of the neutrophil and other mediators in adult respiratory distress syndrome. Mayo Clin Proc 1989;64:1118-32.

8 Sibile Y, Reynolds HY. Macrophages and polymorphonuclear neutrophils in lung defense and injury. $A m$ Rev Respir Dis 1990;141:471-501.

9 Tagan MC, Markert M, Schaller MD, Feihl F, Chiolero R, Perret $\mathrm{CH}$. Oxidative metabolism of circulating granulocytes in adult respiratory distress syndrome. Am $7 \mathrm{Med}$ cytes in adult respiratory dis

10 Glauser MP, Zanetti G, Baumgartner J-D, Cohen J. Septic shock: pathogenesis. Lancet 1991;338:732-6.

11 Cree IA, Michie HJ, Moussa K, Winter JH, Stephens S. Sepsis and shock (letter). Lancet 1991;338:1221-2.

12 Blair AL, Cree IA, Beck JS, Hastings MJG. Measurement of phagocyte chemiluminescence in a microtitre plate format. F Immunol Methods 1988;112:163-8.

13 Blair AL, Cree IA, Beck JS. Measurement of phagocyte chemiluminescence using a microtitre plate luminometer. chemiluminescence using a microt

14 Cree IA. Assays of human phagocyte function using microtitre plate luminometers. In: Stanley PE, Kricka LJ, eds. Bioluminescence and chemiluminescence: current status. Chichester: Wiley, 1991:261-4.

15 Engelberts I, Moller A, Schoen GJM, van der Linden CJ, Buurman WA. Evaluation of measurement of human TNF in plasma by ELISA. Lymphokine Cytokine Res 1991;10:69-76.

16 De Sole P. Polymorphonuclear chemiluminescence: some clinical applications. $\mathcal{F}$ Biolum Chemilum 1989;4:251-62.

17 Ross GD, Cain JA, Lachmann PJ. Membrane complement receptor type three (CR3) has lectin-like properties analogous to bovine conglutinin and functions as receptor for zymosan and rabbit erythrocytes as well as a receptor for iC3b. F Immunol 1985;134:3307-15.

18 Williams JD, Topley N, Alobaidi HM, Harber MJ. Activation of human polymorphonuclear leucocytes by particulate zymosan is related to both its major carbohydrate components: glucan and mannan. Immunology 1986;58:117-24.

19 Sanchez Gascon F, Aviles MJ, Alvarez R, Contessotto C, Ontanon J, Cano A, et al. Soluble interleukin-2 receptors in patients with lung disease. Am Rev Respir Dis 1992;145:A274.

20 Zimmerman GA, Prescott SM, McIntyre TM. Endothelial cell interactions with granulocytes: tethering and signalling molecules. Immunol Today 1992;13:93-100.

21 Hyers TM, Tricomi SM, Dettenmier PA, Fowler AA. Tumour necrosis factor levels in serum and bronchoalveolar lavage fluid of patients with the adult respiratory veolar lavage fluid of patients with the adult respiratory

22 Roten R, Markert M, Feihl F, Schaller MD, Tagan MC, Perret C. Plasma levels of tumor necrosis factor in the adult respiratory distress syndrome. Am Rev Respir Dis 1991;143:590-2.

23 Dinarello CA. Interleukin-1 and interleukin 1 antagonism. Immunol Today 1991;77:1627-52. 